ISSN 1997-5902

\title{
Caractérisation bactériologique des eaux résiduaires brutes de la ville de Cotonou (Bénin)
}

\author{
1Dogbè Clément ADJAHOUINOU*, ${ }^{3}$ Boniface YEHOUENOU, 1,2 Mouhamadou Nourou Dine LIADY et \\ ${ }^{1}$ Emile Didier FIOGBE \\ ${ }^{1}$ Laboratoire de Recherche sur les Zones Humides. Département de Zoologie. Faculté des Sciences et Techniques. \\ Université d'Abomey-Calavi (Bénin). 01BP526 Cotonou Bénin. \\ 2Unité «Assainissement et Environnement». Département Sciences et Gestion de l'Environnement. Université de Liège, \\ Campus d'Arlon. Avenue de Longwy 185, B - 6700 Arlon, Belgique. Tél: 003263230849 - Fax : 003263230800. \\ ${ }^{3}$ Laboratoire d'Étude et de Recherche en Chimie Appliquée, Ecole Polytechnique d'Abomey-Calavi (EPAC), Université \\ d'Abomey-Calavi, Bénin.01BP2009 Cotonou Bénin. \\ *Auteur correspondant : clementiofr@yahoo.fr, Tél: (00229) 97265322
}

Original submitted in on $4^{\text {th }}$ February 2014. Published online at www.m.elewa.org on 30thJune 2014. http://dx.doi.org/10.4314/iab.v78i1.13

\section{RESUME}

Objectif : La présente étude vise à évaluer la pollution fécale des eaux usées de Cotonou, ville la plus peuplée du Bénin.

Méthodologie et Résultats: Trois stations correspondant au point de rejet de trois collecteurs ont été échantillonnées durant une période de 11 mois allant de Janvier à Novembre 2013. La température, le pH, la conductivité électrique et la teneur en oxygène dissous ont été mesurés in situ. Le dénombrement des bactéries indicatrices de contamination fécale a été réalisé par la méthode d'ensemencement par incorporation en milieu nutritif spécifique. Les charges enregistrées pour les différentes bactéries dénombrées ont été hors

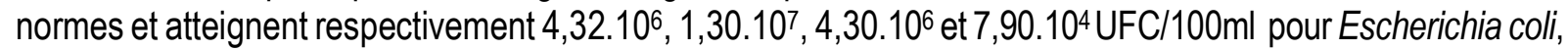
les coliformes totaux, les streptocoques fécaux et les anaérobies sulfito-réducteurs. Les valeurs notées pour les paramètres physico-chimiques ont été aussi irrégulières par rapport aux normes de rejet mais favorables pour la plupart au bon développement des microorganismes.

Conclusions et application : Les eaux résiduaires étudiées sont considérablement chargées en germes fécaux. Ceci indique une importante contamination fécale, source potentielle d'épidémie. Leur épuration est donc nécessaire avant leur rejet dans l'environnement afin de lever la menace qu'elles constituent pour la santé publique.

Mots-clés: pollution fécale, eaux résiduaires, collecteurs, Cotonou.

\section{Bacteriological characterization of raw wastewaters of Cotonou city (Benin) ABSTRACT}

Objective: The current study aims to assess the fecal pollution of the wastewater of Cotonou, the most populated city of Benin.

Methodology and Results: Three stations corresponding to discharge point of three main sewers have been sampled during 11 months from January to November 2013. Temperature, pH, electric conductivity and dissolved oxygen were measured in situ. Counting of fecal contamination indicator bacteria was carried out 

ville de Cotonou (Benin)

through seeding method by incorporation in specific nutritious media. Counts of different bacteria numbered were out of standards and reached respectively $4.32 .10^{6}, 1.30 .10^{7}, 4.30 .10^{6}$ and $7.90 .10^{4} \mathrm{UFC} / 100 \mathrm{ml}$ for Escherichia coli, total coliforms, fecal streptococci and anaerobic sulphite reducers. Physico-chemical parameters values were not likewise up to standards of pouring but favourable, for the most, to suitable growth of microorganisms.

Conclusions and application: Studied wastewaters are high loaded in fecal germs. This indicates an important fecal contamination, potential epidemic source. Their purification appears necessary before pouring in environment in order to raise the threat which they constitute for people's health.

Key-words: fecal pollution, wastewaters, main sewers, Cotonou.

\section{INTRODUCTION}

L'eau, denrée essentielle et indispensable pour la vie, peut également constituer une source de maladies lorsqu'elle est contaminée par des agents potentiellement pathogènes (Hassoune et al., 2010 ; el Haissoufi et al., 2011). Cette contamination est généralement la conséquence de son utilisation dans les différentes activités humaines (domestiques, agricoles, industrielles) qui génèrent de grandes quantités d'eaux usées pouvant contenir une multitude de microorganismes pathogènes tels que des bactéries, des virus et des parasites (Leclerc et al., 1982 ; Shuval et al., 1986 ; USEPA, 2004). Ainsi, la réutilisation de ces eaux résiduaires ou leur rejet sans aucun traitement dans le milieu, comme c'est le cas dans nombre de pays en développement (Ajuzie et Osaghae, 2012), crée des problèmes d'hygiène, sources de propagation de maladies hydriques, à l'origine d'un taux de mortalité élevé des populations dans ces pays (Bou Saad et al., 2007 ; Hamaidi et al., 2009). Le Bénin, à l'instar de plusieurs autres pays en développement, n'est pas à l'abri de ces problèmes de santé publique liés au non assainissement des eaux usées. En effet, les taux de prévalence des maladies diarrhéiques $(2 \%$

\section{MATERIEL ET METHODES}

Milieu d'étude : La présente étude a été conduite à Cotonou, capitale économique et ville la plus peuplée du Bénin située entre $6^{\circ} 20^{\prime}$ et $6^{\circ} 23^{\prime}$ de latitude Nord et $2^{\circ} 22^{\prime}$ et $2^{\circ} 30^{\prime}$ de longitude Est (Figure 1). Limité à l'ouest par la commune d'Abomey-Calavi et à l'est par celle de SèmèKpodji, Cotonou dispose de deux frontières naturelles reliées par un chenal (lagune de Cotonou) : le lac Nokoué au Nord et l'océan Atlantique au sud. Long d'environ 4,5 $\mathrm{km}$, la lagune constitue l'exutoire de nombreux caniveaux et collecteurs érigés dans la ville, normalement, pour des causes principales de décès) et gastrointestinales, potentiellement hydriques, ont été respectivement estimés à $2,8 \%$ et $6,5 \%$ de la situation épidémiologique au Bénin en 2012 (SNIGSMS, 2013). A Cotonou, ville la plus peuplée de ce pays, la grande quantité d'eaux usées issues des différents usages de l'eau, est déversée sans traitement soit dans la nature ou dans la cour des maisons ( $83,8 \%$ de la population) ou dans les canaux ou caniveaux fermés ou à ciel ouvert $(5,3 \%$ de la population) érigés pour endiguer normalement les eaux pluviales (INSAE, 2003). La plupart de ces collecteurs rejettent leur contenu dans la lagune de Cotonou reliant le lac Nokoué et l'océan Atlantique, plans d'eau fortement exploités par la population pour la pêche, le transport de personnes et de marchandises, la baignade. La présente étude a été entreprise dans le but de faire une évaluation de la pollution fécale des eaux usées de la ville de Cotonou par l'analyse des bactéries indicatrices (Escherichia coli, coliformes totaux, streptocoques fécaux, anaérobie sulfito-réducteur) inféodées à ces eaux, afin d'apprécier les risques de leur rejet sans traitement dans l'environnement.

assainir les eaux pluviales, mais qui reçoivent en permanence des eaux résiduaires brutes émanant des activités humaines. La zone d'étude, en raison de sa situation géographique, est soumise à un climat subéquatorial caractérisé par deux saisons pluvieuses (une grande d'avril à juillet et une petite marquée par la crue des eaux continentales allant d'octobre à novembre) et deux saisons sèches, la grande couvrant les mois de décembre à mars et la petite s'étendant sur les mois d'août et de septembre (Adam et Boko, 1993). 

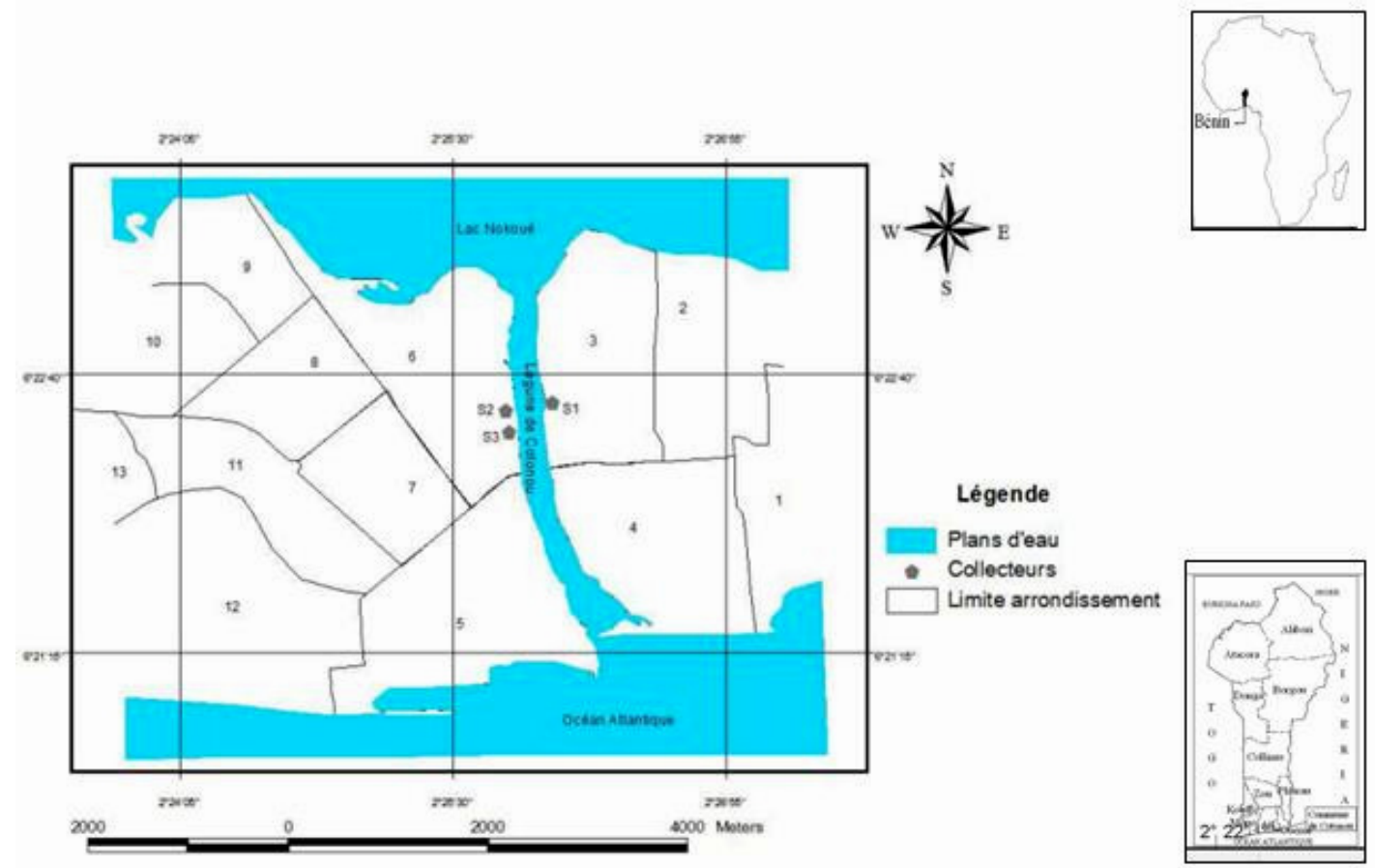

Figure 1 : Carte de la ville de Cotonou montrant les stations d'échantillonnage

Echantillonnage et conservation des échantillons : Dans cette étude, trois collecteurs d'eau déversant leur contenu dans le chenal de Cotonou, ont été choisis, en tenant compte, entre autres, de leur longueur et de la pression anthropique dont ils font l'objet (Tableau 1). En vue d'avoir des échantillons représentatifs, trois stations correspondant chacune au point de rejet d'un des collecteurs, ont été échantillonnées (Figure 1 et Tableau 1). Les campagnes d'échantillonnage ont été réalisées mensuellement pendant 11 mois de janvier à Novembre
2013 afin de couvrir toutes les saisons de l'année. Au total, 33 échantillons d'eau ont été ainsi prélevés suivant les recommandations requises pour les analyses microbiologiques (Rodier et al., 2009). Des bouteilles stériles et lestées ont été utilisées, afin de prélever des échantillons représentatifs de toute la colonne d'eau ( $\mathrm{du}$ fond à la surface). Les échantillons ainsi recueillis ont été conservés au frais dans une glacière jusqu'à leur analyse au laboratoire 6 heures maximum après leur prélèvement.

Tableau 1 : Caractéristiques des collecteurs et localisations géographiques des stations d'échantillonnage

Coordonnées

Collecteurs Longueur Quartiers ou zones desservis Stations géographiques

\begin{tabular}{|c|c|c|c|c|c|}
\hline & & & & Latitude & Longitude \\
\hline T1 & $1 \mathrm{~km}$ & Midombo et environs & S1 & $06^{\circ} 22^{\prime} 38^{\prime \prime} \mathrm{N}$ & $002^{\circ} 26^{\prime} 18^{\prime \prime} \mathrm{E}$ \\
\hline B & $2 \mathrm{~km}$ & Gbéto et Marché Dantokpa & S2 & $06^{\circ} 22^{\prime} 34^{\prime \prime} \mathrm{N}$ & $002^{\circ} 26^{\prime} 06^{\prime \prime} \mathrm{E}$ \\
\hline A & $4 \mathrm{~km}$ & $\begin{array}{l}\text { Hindé, Djidjè, Aïdjèdo, Jéricho et } \\
\text { Gbégamey }\end{array}$ & S3 & $06^{\circ} 22^{\prime} 55^{\prime \prime} \mathrm{N}$ & $002^{\circ} 26^{\prime} 02^{\prime \prime} \mathrm{E}$ \\
\hline
\end{tabular}

Analyses physico-chimiques et bactériologiques : A chaque échantillonnage, certains paramètres physico- chimiques ont été mesurés in situ. II s'agit de la température, du pH, de la conductivité électrique avec un 

ville de Cotonou (Benin)

conductimètre multifonction portable WTWpH/cond 340i/SET et de la teneur en oxygène dissous à l'aide d'un oxymètre portable WTW oxi 340i/SET. Le dénombrement des bactéries indicatrices de pollution d'origine fécale a été fait par ensemencement de $1 \mathrm{~mL}$ de différente dilution des échantillons $\left(10^{-1}, 10^{-2}, 10^{-3}\right)$ par incorporation dans une gélose nutritive en surfusion à $45^{\circ} \mathrm{C}$ (Rodier et al., 2009). Ainsi, La gélose sélective Brilliance E.coli/coliform (Oxoid, CM 1046) a été utilisée pour dénombrer les coliformes totaux, par la détection simultanée d'E. coli (colonies bleues) et des autres coliformes (colonies rouges) en boîte de pétri, après $24 \mathrm{~h}$ d'incubation à $37^{\circ} \mathrm{C}$. Les streptocoques fécaux ont été dénombrés par ensemencement (colonies rouges) dans la gélose de Slanetz et Bartley (Oxoid, CM 0377) en boîte de pétri, après $48 \mathrm{~h}$ d'incubation à $37^{\circ} \mathrm{C}$. La gélose viande-foie (Oxoid, CM 0918) a servi pour l'estimation des spores d'anaérobies sulfito-réducteurs en tube à essai (colonies entourées d'halo noir), après destruction des formes végétatives à $80^{\circ} \mathrm{C}$, suivie de $24 \mathrm{~h}$ ou $48 \mathrm{~h}$ d'incubation à $37^{\circ} \mathrm{C}$.

\section{RESULTATS}

Paramètres physico-chimiques: Les valeurs moyennes des paramètres physico-chimiques mesurés au niveau de chaque station sont consignées dans le Tableau 2. Le pH et la teneur en oxygène dissous des eaux usées n'ont pas significativement variés d'une station à une autre $(P>0,05)$. En revanche, une différence
Analyses statistiques : Afin de comparer les résultats des trois stations entre eux, une analyse de variance à un facteur (ANOVA1) a été effectuée pour chaque paramètre, après vérification de la normalité (test de Shapiro-Wilk) et de l'homoscédasticité (Test de Bartlett) des données (Scherrer, 2007). Ce test a été suivi en cas de nécessité, du test de Turkey pour déterminer les différences significatives entre les stations prises deux à deux. Lorsque les conditions de normalité et d'homoscédasticité ne sont pas remplies, le test non paramétrique de Kruskall-Wallis a été utilisé, suivi au besoin, du test de Mann-Whitney pour ressortir les différences significatives entre les stations. En outre, le coefficient de corrélation de Pearson ( $r$ ) a été calculé pour vérifier la corrélation qui existerait entre les paramètres physico-chimiques et bactériologiques (Snedecor et Cochram, 1962 ; Adingra et al., 2012).

Ces différentes analyses statistiques ont été réalisées avec le logiciel statistique Minitab14.

significative a été notée entre les stations, pour la température et la conductivité électrique des eaux $(P<0,05)$. En effet, les valeurs moyennes notées pour ces deux paramètres à la station $\mathrm{S} 1$, ont été respectivement plus élevée et plus faible que celles enregistrées au niveau des deux autres stations.

Tableau 2: Valeurs Moyennes suivies d'écartype des paramètres physico-chimiques des eaux usées de Cotonou (Temp : Température ; CE : Conductivité électrique ; OD : Oxygène dissous)

\begin{tabular}{ccccc}
\hline \multirow{2}{*}{ Stations } & \multicolumn{4}{c}{ Paramètres } \\
\cline { 2 - 5 } & $\left.\operatorname{Temp}^{\circ} \mathrm{C}\right)$ & $\mathrm{pH}$ & $\mathrm{CE}(\mu \mathrm{S} / \mathrm{cm})$ & $\mathrm{OD}(\mathrm{mg} / \mathrm{l})$ \\
\hline S1 & $33,45 \pm 2,86$ a & $5,63 \pm 0,86$ a & $1142 \pm 1154$ a & $2,78 \pm 2,39$ a \\
S2 & $30,38 \pm 1,62 \mathrm{~b}$ & $5,70 \pm 0,93$ a & $5150 \pm 3463 \mathrm{~b}$ & $2,15 \pm 1,77$ a \\
S3 & $29,03 \pm 0,99^{\mathrm{b}}$ & $6,08 \pm 0,77^{\mathrm{a}}$ & $3320 \pm 1671^{\mathrm{b}}$ & $2,64 \pm 2,31$ a \\
\hline
\end{tabular}

Dans la même colonne, les moyennes suivies de lettres différentes sont significativement différentes $(P<0,05)$.

Paramètres microbiologiques : Les charges moyennes en germes fécaux enregistrées aux différentes stations d'échantillonnages sont présentées dans le Tableau 3. Aucune différence significative $(P>0,05)$ n'a été observée entre les charges microbiennes notées au niveau des différentes stations sauf celle des streptocoques fécaux qui s'est révélée significativement plus importante au niveau de la station $2(P<0,05)$. 

ville de Cotonou (Benin)

Tableau 3 : Charges bactériennes moyennes (UFC/100mL) dans les eaux usées de Cotonou (Ec: E. coli; CT: coliformes totaux ; SF : streptocoques fécaux ; ASR: anaérobies sulfito-réducteurs)

\begin{tabular}{ccccc}
\hline \multirow{2}{*}{ Stations } & & \multicolumn{3}{c}{ Charges bactériennes (UFC/100 ml) } \\
\cline { 2 - 5 } & Ec & CT & SF & ASR \\
\hline S1 & $5,39.10^{5} \pm 2,30.10^{5} \mathrm{a}$ & $2,35.10^{6} \pm 1,84.10^{6} \mathrm{a}$ & $1,03.10^{6} \pm 7,18.10^{5}$ ab & $4,44.10^{4} \pm 2,38.10^{4} \mathrm{a}$ \\
S2 & $9,85.10^{5} \pm 1,18.10^{6} \mathrm{a}$ & $3,87.10^{6} \pm 3,70.10^{6} \mathrm{a}$ & $1,29.10^{6} \pm 1,09.10^{6} \mathrm{a}$ & $3,80.10^{4} \pm 1,98.10^{4} \mathrm{a}$ \\
& & & & \\
S3 & $3,85.10^{5} \pm 2,27.10^{5} \mathrm{a}$ & $1,67.10^{6} \pm 1,26.10^{6} \mathrm{a}$ & $5,77.10^{5} \pm 4,05.10^{5 \mathrm{~b}}$ & $3,21.10^{4} \pm 1,69.10^{4} \mathrm{a}$ \\
\hline
\end{tabular}

Dans la même colonne, les moyennes suivies de lettres différentes sont significativement différentes $(P<0,05)$.

II convient néanmoins de noter que les densités en germes microbiens varient entre $1,01.10^{5}$ UFC/100mL (S3) et $4,32.10^{6} \mathrm{UFC} / 100 \mathrm{~mL}$ (S2) pour E. coli (Figure 2), $4,20.10^{5}$ UFC/100mL (S1) et $1,30.10^{7}$ UFC/100mL (S2) pour les coliformes totaux (Figure 3),
$1,95.10^{5} \mathrm{UFC} / 100 \mathrm{~mL}$ (S3) et $4,30.10^{6} \mathrm{UFC} / 100 \mathrm{~mL}$ (S2) pour les streptocoques fécaux (Figure 4 ) et entre $5,00.10^{3}$ (S3) et 7,90.104 UFC/100mL (S1) pour les Anaérobies sulfito-réducteurs (Figure 5).

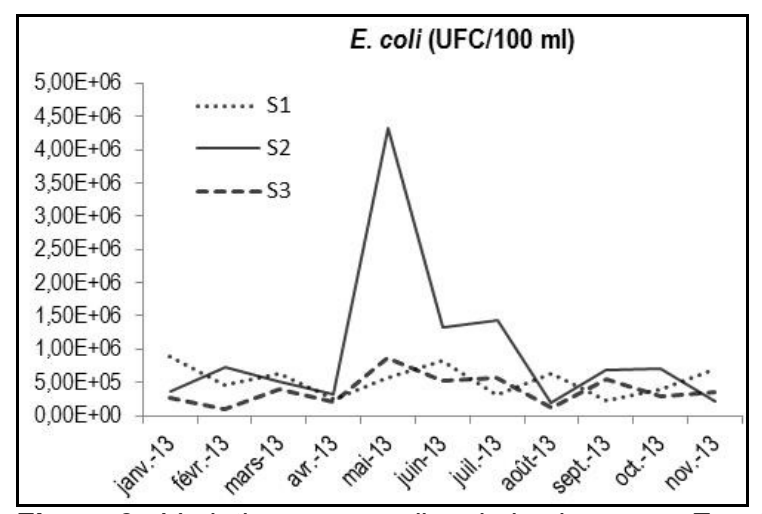

Figure 2 : Variations mensuelles de la charge en $E$. coli

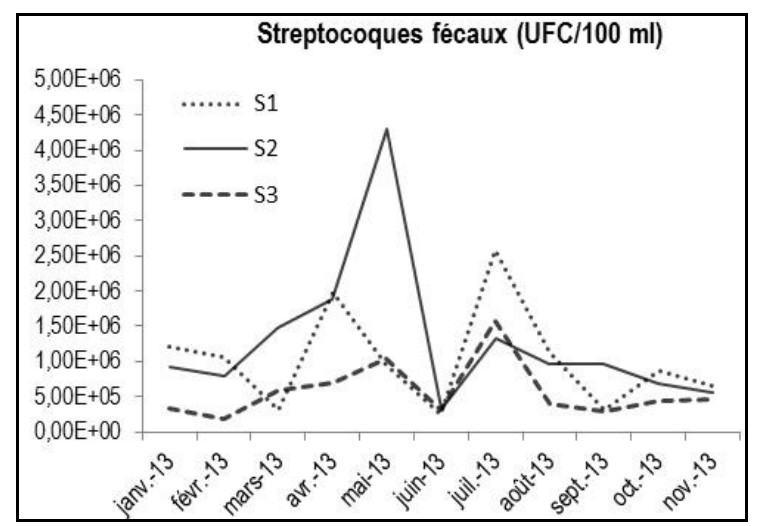

Figure 4: Variations mensuelles de la charge en streptocoques fécaux

La matrice de corrélation entre la population bactérienne et les variables abiotiques (Tableau 4) montre que la

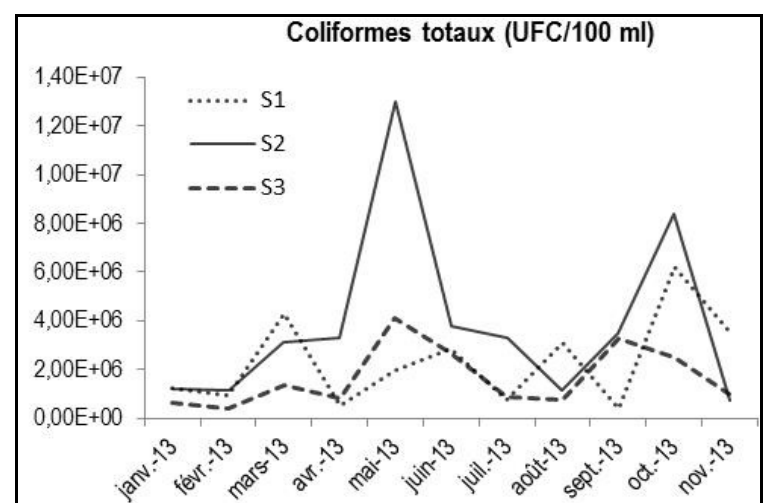

Figure 3 : Variations mensuelles de la charge en coliformes totaux

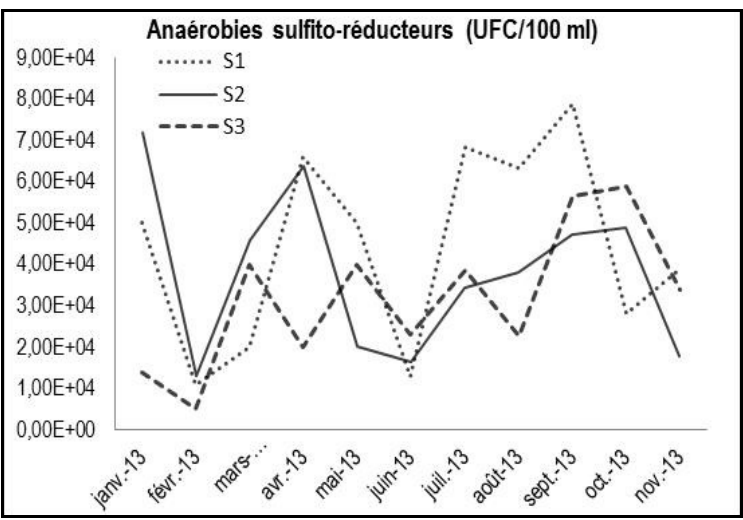

Figure 5: Variations mensuelles de la charge en anaérobies sulfito-réducteurs

conductivité électrique est significativement corrélée positivement avec la charge en $E$. coli $(r=0,404$; 

ville de Cotonou (Benin)

$\mathrm{P}<0,05)$ et négativement avec la population d'anaérobie sulfito-réducteurs $(r=-0,420 ; P<0,05)$. En outre, une corrélation positive hautement significative a été notée entre $E$. coli et les coliformes totaux $(r=0,790 ; P<0,001)$,
E coli et les streptocoques fécaux $(r=0,664 ; P<0,001)$ et entre les coliformes totaux et les streptocoques fécaux ( $r$ $=0,483 ; P<0,01)$.

Tableau 4 : Matrice de corrélation des paramètres physico-chimiques avec les charges bactériennes ( $\mathrm{N}=33$ échantillons)

\begin{tabular}{|c|c|c|c|c|c|c|c|c|}
\hline Paramètres & Ec & CT & SF & ASR & Temp & $\mathrm{pH}$ & CE & OD \\
\hline Ec & 1 & & & & & & & \\
\hline CT & $0,790^{* * *}$ & 1 & & & & & & \\
\hline SF & $0,664^{\star \star *}$ & $0,483^{* *}$ & 1 & & & & & \\
\hline ASR & $-0,194$ & $-0,086$ & 0,216 & 1 & & & & \\
\hline Temp & 0,084 & $-0,035$ & 0,062 & 0,038 & 1 & & & \\
\hline $\mathrm{pH}$ & 0,013 & 0,184 & $-0,032$ & 0,108 & $-0,052$ & 1 & & \\
\hline CE & $0,404^{*}$ & 0,267 & 0,193 & $-0,420^{*}$ & $-0,191$ & 0,293 & 1 & \\
\hline OD & 0,268 & 0,083 & 0,176 & 0,157 & $-0,185$ & $-0,170$ & $-0,138$ & 1 \\
\hline
\end{tabular}

\section{DISCUSSION}

Les températures moyennes enregistrées pour les eaux étudiées sont proches du seuil de $30^{\circ} \mathrm{C}$ généralement indiqué pour le rejet des eaux résiduaires (DEEC, 2001; Journal officiel de la République algérienne, 2003) au niveau des stations S1 et S2. Ce seuil est toutefois dépassé à la station 3 . Ces températures, étant supérieures à $15^{\circ} \mathrm{C}$, sont favorables au développement des microorganismes (Rodier et al., 1996) tout comme celles des eaux résiduaires domestiques d'AbomeyCalavi (Aïna et al., 2012), ville frontalière de Cotonou qui sont néanmoins plus faibles. Le pH moyen noté pour ces eaux est acide à toutes les stations contrairement aux résultats de Abouelouafa et al. (2002) et N'Diaye et al. (2011), qui ont enregistré des $\mathrm{pH}$ légèrement à moyennement alcalins, respectivement pour les eaux résiduaires des villes d'Oujda au Maroc et de Nouakchott en Mauritanie. Ce caractère des eaux résiduaires de Cotonou, ne répond pas aux normes de l'Organisation Mondiale de la Santé qui a fixé le pH entre 6 à 9 pour les rejets d'eau dans l'environnement (OMS, 2006). En outre, les $\mathrm{pH}$ enregistrés étant compris entre 5 et 8,5 , sont favorables à la croissance et à la survie des microorganismes (Mara, 1980). Pour ce qui concerne la conductivité électrique, les valeurs enregistrées témoignent d'une minéralisation excessive $(>1000 \mu \mathrm{s} / \mathrm{cm})$ des eaux (Genoud et al., 2007) et surpassent ainsi la valeur limite de rejet en milieu naturel $(2500 \mu \mathrm{s} / \mathrm{cm})$ (MATEE, 2005). Ces résultats sont conformes à ceux rapportés par Abouelouafa et al, (2002), N'Diaye et al.(2011), et El Hachemi et al. (2012).

L'analyse microbiologique des eaux des trois collecteurs prospectés a révélé qu'elles possèdent des charges bactériennes largement au-dessus du seuil de $10^{3}$ UFC/ $100 \mathrm{ml}$ fixé par l'OMS (1989) pour le rejet direct dans l'environnement. Ce même constat a été fait par plusieurs auteurs ayant menés des travaux similaires (Abouelouafa et al, 2002 ; Fagrouch et al, 2010 ; N'Diaye et al, 2011 ; Hamdi et al, 2012). La forte densité des E. coli, bactéries indicatrices les plus spécifiques d'une pollution fécale (CEAEQ, 2000 ; Edberg et al., 2000), dans les eaux analysées, indique clairement leur contamination par des germes fécaux et par conséquent, le risque épidémiologique potentiel que représente leur rejet sans traitement (Servais et al., 2006). En effet, la présence de ces entérobactéries, quoique non pathogènes dans leur grande majorité, doit faire sérieusement soupçonner celle 

ville de Cotonou (Benin)

de plusieurs autres microorganismes pathogènes (Elmund et al, 1999 ; Ajit et al., 2009). II n'en demeure pas moins pour les coliformes totaux et les streptocoques fécaux qui constituent autant que les $E$. coli, des indicateurs principaux d'une contamination fécale (Servais et al., 2006). Ceci se justifie par la corrélation positive hautement significative notée entre ces entérobactéries. La recherche d'un de ces microorganismes permettrait donc de prédire la dynamique des autres et par conséquent de juger la qualité bactériologique de leur biotope. II convient toutefois de noter que les charges enregistrées pour les coliformes totaux et les streptocoques fécaux sont plus faibles que celles des eaux brutes de la ville d'Oujda (Abouelouafa et al., 2002) et dépassent largement celles enregistrées pour les eaux usées de Nouakchott (N'Diaye et al., 2011) et d'Ouargla (Hamdi et al., 2012). Cette disparité serait due aux spécificités liées soit aux paramètres physico-chimiques de ces eaux, soit à leur origine (domestique ou industrielle). La charge importante en anaérobies sulfito-réducteurs des eaux étudiées se

\section{CONCLUSIONS}

Les résultats de cette étude montrent que les effluents bruts de la ville de Cotonou véhiculent d'importantes charges hors normes de bactéries, témoins de pollution fécale considérable. Le déversement sans traitement de ces eaux résiduaires, constitue sans nul doute, une menace pour la santé des populations riveraines et justifierait par la faible teneur en oxygène dissous notée pour ces eaux, ce qui témoigne de leur caractère quasianaérobie (IBGE, 2005). Ce caractère des eaux confirme les résultats obtenus par Adjahouinou et al. (2012) pour les eaux du collecteur de Dantokpa (Cotonou) débouchant sur la station 2. La concentration en spores d'anaérobies sulfito-réducteurs est, pour ces mêmes raisons, moins importante que celle rapportée par Fagrouch et al. (2010) dans les eaux usées brutes de la ville de Taourirt (Maroc) qui sont en effet, plus pauvre en oxygène dissous. Dans le groupe des anaérobies sulfitoréducteurs figurent les Clostridium, notamment Clostridium perfringens, microorganisme d'origine fécale dont les spores sont très résistantes dans l'environnement (Sorensen et al., 1989 ; Davies et al., 1995). Ces bactéries constituent de ce fait, les indicateurs les plus appropriés des agents pathogènes les plus résistants de l'eau tels que les virus entériques, les protozoaires parasites et les œufs d'helminthes (Payment et Franco, 1993).

usagères des exutoires dont la lagune de Cotonou. II va falloir impérieusement entreprendre leur assainissement et leur épuration avant tout rejet en milieu naturel afin de prévenir les épisodes épidémiologiques dus aux maladies hydriques récurrentes dans la ville surtout en saisons pluvieuses, périodes de forte insalubrité.

\section{REMERCIEMENTS}

Cette étude a été financée par le Ministère d'Etat Chargé de l'Enseignement Supérieur et de la Recherche Scientifique de la République du Bénin, qui a octroyé une allocation de thèse de doctorat à Clément Dogbè Adjahouinou.

\section{REFERENCES BIBLIOGRAPHIQUES}

Abouelouafa M, El Halouani H, Kharboua M, Berrichi A, 2002. Caractérisation physico-chimique et bactériologique des eaux usées brutes de la ville d'Oujda: canal principal et Oued Bounaïm. Actes Inst. Agron. Vet. (Maroc) 22 (3):143-150.

Adam KS et Boko M, 1993. Le Bénin. Les Editions du Flamboyant / EDICEF. Vanves, Cedex. 96pp.

Adingra AA, Kouadio AN, Blé MC, Kouassi AM, 2012. Bacteriological analysis of surface water collected from the Grand-Lahou lagoon, Côte d'ivoire. African Journal of Microbiology Research 6 (13): 3097-3105.

Adjahouinou DC, Liady ND, Fiogbé ED, 2012. Diversité phytoplanctonique et niveau de pollution des eaux du collecteur de Dantokpa (CotonouBénin). Int. J. Biol. Chem. Sci. 6 (5): 1938-1949.

Aina MP, Djihouessi B, Vissin EW, Kpondjo NM, Gbèdo V, Sohounhloué KCD, 2012. Characterization of the domestic wastewaters and dimensionality of a pilot treatment station by lagooning at AbomeyCalavi city-Benin. J. Engineering Sci.1 (1): 45-50.

Ajit KP, Bhaskar CA, Anil M (2009). Occurrence and distribution of bacterial pathogens in coastal Indicators and waters of Orissa. Indian. J. Mar. Sci. 38 (4): 474-480.

Ajuzie CU et Osaghae BA, 2012. The bacterial and physico-chemical properties of hair salon wastewater and contaminated soil in Benin 
metropolis. African Journal of Biotechnology 10 (11): 2066-2069.

Bou Saab H, Nassif N, El Samrani AG, Rosette D, Medawar S, Ouaïni N, 2007. Suivi de la qualité bactériologique des eaux de surface (Rivière Nahr Ibrahim, Liban). Revue des Sciences de l'Eau 20 (4): 341-352.

CEAEQ, 2000. Recherche et dénombrement des coliformes fécaux; méthode par filtration sur membrane. Centre d'Expertise en Analyse Environnementale, Gouvernement du Québec. $24 \mathrm{pp}$.

Davies CM, Long JA, Donald M, Ashbolt NJ, 1995. Survival of fecal microorganisms in aquaticsediments of Sydney, Australia. Appl. Environ. Microbiol. 61: 1888-1896.

DEEC (Direction de l'Environnement et des Etablissements Classés)/Sénégal, 2001. Eaux usées : Normes de rejets. Norme sénégalaise NS 05-061Juillet 2001. 27pp.

Edberg, SC, Rice EW, Karlin RJ, Allen MJ, 2000. Escherichia coli: the best biological drinking water indicator for public health protection. Journal of Applied Microbiology 88: 106S-116S.

El Hachemi O, El Halouani H, Meziane M, Torrens A, Salgot M, Sbaa M, 2012. Etude des performances épuratrices dans une station de traitement des eaux usées par lagunage en climat désertique (oasis de figuig - maroc) : aspect bactérien et organique. Rev. Microbiol. Ind. San et Environn. 6 (1): 84-97.

El haissoufi $\mathrm{H}$, Berrada S, Merzouki M, Aabouch M, Bennani L, Benlemlih M, Idir M, Zanibou A, Bennis Y, El OualiLalami A, 2011. Pollution des eaux de puits de certains quartiers de la ville de Fès, Maroc. Rev. Microbiol. Ind. San et Environn 5 (1): 7-68.

Elmund, GK, Allen MJ, Rice EW, 1999. Comparison of Escherichia coli, total coliform and fecal coliform populations as indicators of wastewater treatment efficiency. Water Environ. Res. 71: 332-339.

Fagrouch A, Amyay S, Berrahou A, El Halouani $H$, Abdelmoumen H, 2010. Performances d'abattement des germes pathogènes en lagunage naturel sous climat aride: cas de la filière de traitement des eaux usées de la ville de Taourirt. Afrique SCIENCE 6 (3): 87 - 102.

Genoud L, Bagnoud R, Baudin MN, Furrer P, Venetz PM, 1997. Etude du réseau de drainage de la plaine de Vionnaz. Service de la Protection de L'Environnement et Laboratoire Cantonal du Canton du Valais. 85 pp.

Hamaidi MS, Hamaidi F, Zoubiri A, Benouaklil F, Dhan Y, 2009. Etude de la dynamique des populations phytoplanctoniques et résultats préliminaires sur les blooms toxiques à cyanobacteries dans le barrage de Ghrib (Ain Defla-Algérie). European Journal of Scientific Research 32 (3): 369-380.

Hamdi W, Youcefi M, Touil Y, Bougrinat R, Ferhi N, Ould El Hadj MD, 2012. Contribution à l'étude de quelques caractéristiques physico chimiques et hygiéniques des eaux usées issues de rejets de certaines localités de la cuvette de Ouargla (Sahara septentrional Est algérien): impact sur le milieu récepteur. Algerian journal of arid environment 2 (1): 56-63.

Hassoune E, El Kettani S, Koulali Y, Bouzidi A, 2010. Contamination bactériologique des eaux souterraines par les eaux usées de la ville de Settat, Maroc. Rev. Microbiol. Ind. San et Environn. 4 (1): 1-21.

IBGE (Institut Bruxellois pour la Gestion de l'Environnement), 2005. Qualité physicochimique et chimique des eaux de surface: cadre général. Les données de l'IBGE : "L'eau à Bruxelles". 16pp. www.ibgebim.be consulté le 19 novembre 2013

INSAE (Institut National de la Statistique et de l'Analyse Economique)/Bénin, 2003: Troisième Recensement Général de la population et de l'Habitation de 2002. Synthèse des résultats, octobre 2003. 35pp.

Journal officiel de la République algérienne, 2003. Normes de rejets dans le milieu récepteur, 46 , 7-12.

Leclerc H., Festy B., Lazar P., 1982. Connaissance actuelle de la pathologie hydrique. Rev. Epidemiol. santé publique 30 (3): 363-385.

Mara D, 1980. Sewage treatment in hot climates. Ed. John Willey \& sons. $168 \mathrm{pp}$.

MATEE (Ministère de l'Aménagement, du Territoire, de l'Eau et de l'Environnement)/Royaume du Maroc, 2005. Décret $n^{\circ}$ 2-04-553 24 janvier 2005 relatif aux déversements, écoulements, rejets, dépôts directs ou indirects dans les eaux superficielles ou souterraines.

N'diaye AD, Kankou MOSAO, Lo B, Namr KI, 2011. Caractérisation de la pollution bactériologique des effluents de la ville de Nouakchott, irrigués 
dans le périmètre maraîcher de Sebkha. Int. J. Biol. Chem. Sci. 5 (2): 748-754.

OMS (Organisation mondiale de la santé), 1989. L'utilisation des eaux usées en agriculture et en aquaculture : recommandations à visées sanitaires. Rapport technique $n^{\circ} 778$, Genève.

OMS (Organisation mondiale de la santé), 2006. Guidelines for the safe use of wastewater, excreta and greywater. World Health Organization. Geneva. 36pp.

Payment $P$ et Franco $E$, 1993. Clostridium perfringens and somatic coliphages as indicators of the efficiency of drinking water treatment for viruses and protozoan cysts. Appl. Environ. Microbiol. 59, 2418-2424.

Rodier J, Bazin C, Broutin JP, Chambon P, Champsaur H, Rodier L, 1996. L'Analyse de l'Eau. 8è édition. Dunod : Paris. 1384 pp.

Rodier J, Legube B, Merlet N, 2009. L'Analyse de l'Eau. 9è édition. Dunod : Paris. 1579 pp.

Scherrer B, 2007. Biostatistique, Volume 1. Gaëtan Morin Ed., Boucherville. xiv $+816 \mathrm{pp}$.

Servais P, Garcia-Armisen T, Lizin P, Mercier P, Anzil A, 2006. Modélisation de la dynamique des indicateurs de qualité microbiologique en estuaire de Seine. Rapport scientifique SeineAval 3.11pp.

Shuval HI, Adin A, Fattal B, Rawitz E, Yekutiel P, 1986. Wastewater irrigation in developing countries. World Bank Publ. Tech. paper series $n^{\circ} 51$.

Snedecor GW et Cochram WG, 1962. Factorial Experiments. In Statistical methods- Oxford and IBM Publishing Co. Calcutta, 339-380.

SNIGS-MS (Système National d'Information et de Gestion Sanitaires - Ministère de la Santé)/Bénin, 2013. Annuaire des Statistiques Sanitaires année 2012. 147pp.

Sorensen DL, Eberl SG et Diksa RA, 1989. Clostridium perfringens as a point source indicator in nonpoint-polluted streams. Wat. Res. 23: 191-197.

USEPA (United States Environmental Protection Agency), 2004. Guidelines for water reuse. EPA/625/R-04/108. 480pp. 